\title{
ON EXTERIOR DERIVATIVES AND SOLUTIONS OF ORDINARY DIFFERENTIAL EQUATIONS
}

\author{
BY \\ PHILIP HARTMAN( ${ }^{(1)}$
}

Part I is concerned with sufficient conditions for the local uniqueness of solutions of nonlinear systems of ordinary differential equations and with necessary and sufficient conditions for the $C^{1}$ character of general solutions. Parts II and III give applications of these results.

It is shown, in Part II, that the theorems of Part I imply those of $[4 ; 8]$ on the local uniqueness of geodesics and on the introduction of geodesic coordinates for a binary positive definite Riemannian metric. In fact, Part I makes possible the extensions of these theorems from 2 to $n$ dimensions and from geodesics to solutions of more general Euler-Lagrange systems.

In Part III, the theorems of Part I are used to prove a theorem of Frobenius, dealing with systems of Pfaffians, under relaxed conditions of differentiability.

\section{General systems}

1. All variables and functions below are real-valued. The letter $s$ represents a real variable, a prime denotes differentiation with respect to $s$. Also, $x$ is an $n$-vector and $z$ is an $m$-vector; correspondingly, $|x|$ or $|z|$ is the $n$-or $m$-dimensional (Euclidean) length of $x$ or $z$. Tensor summation convention will be followed; Latin indices $i, j, k$ vary from 1 to $n$ and Greek indices $\mu, \nu$ go from 1 to $m$.

A linear differential form or Pfaffian in $d x^{1}, \cdots, d x^{n}$, say $\omega=p_{j}(x) d x^{j}$, with coefficients $p_{j}(x) \equiv p_{j}\left(x^{1}, \cdots, x^{n}\right)$, defined on an $x$-domain $D$, is said to be continuous [or of class $C^{k}$ ] if the coefficients are continuous [or of class $C^{k}$ ] on $D$. A continuous differential form $\omega$ is said to possess a continuous [or bounded] exterior derivative $d w$ if there exists a quadratic form in $d x^{1}, \cdots, d x^{n}$, say, $d \omega=r_{j k}(x) d x^{j} d x^{k}$, with coefficients $r_{j k}=-r_{k j}$ continuous [or bounded and measurable] on $D$ and satisfying Stokes' formula

$$
\int_{J} \omega=\iint_{E} d \omega
$$

Received by the editors July 1, 1957.

(1) This research was supported by the United States Air Force through the Air Force Office of Scientific Research of the Air Research and Development Command, under contract No. AF 18(603)-41. Reproduction in whole or in part is permitted for any purpose of the United States Government. 
for every piece of 2-dimensional $C^{1}$-surface $E$ in $D$ bounded by a $C^{1}$-piecewise Jordan curve $J$ in $D$; cf. [1, pp. 65-71].

The following criterion for the local uniqueness of solutions of nonlinear systems of ordinary differential equations will be proved below.

(I) Let $f=f(x, s)$ be a continuous n-vector function defined for $|x|<1$, $|s|<1$. A sufficient condition for the (local) uniqueness of a solution of the initial value problem

$$
x^{\prime}=f(x, s) \text { and } \quad x(0)=0
$$

is that there exist a continuous $n$ by $n$ matrix function $A=A(x, s)$ on $|x|<\epsilon$, $|s|<\epsilon$ for some $\epsilon>0$ such that

$$
\operatorname{det} A \neq 0
$$

and that the $n$ Pfaffians in $d x^{1}, \cdots, d x^{n}, d s$,

$$
\omega=A(d x-f d s)=A d x-b d s, \text { where } b=A f,
$$

have bounded exterior derivatives on $|x|<\epsilon,|s|<\epsilon$.

This generalizes a corresponding theorem for the case $n=1$ in [6, Part I]. If $f=\left(f^{1}, \cdots, f^{n}\right), b=\left(b_{1}, \cdots, b_{n}\right)$, and $A=\left(a_{j k}\right)$, then $\omega$ in (4) represents $n$ linear differential forms $\omega=\left(\omega_{1}, \cdots, \omega_{n}\right)$, where

$$
\omega_{j}=a_{j k} d x^{k}-b_{j} d s,
$$

$b_{j}=a_{j k} f^{k}$ and $j, k=1, \cdots, n$.

(I) is an analogue of the standard condition that $f$ satisfy a Lipschitz condition with respect to $x$ (and is a generalization of that condition when $n=1)$. One can modify (I) to obtain analogues of the Osgood or Nagumo conditions by requiring, for example, that the continuous forms (4) have bounded exterior derivatives on $|x|<\epsilon, \theta \epsilon<|s|<\epsilon$, for every positive $\theta<1$ with bounds $M(\theta)$ for the coefficients of $d \omega$ satisfying $M(\theta)=o(1 / \theta)$ as $\theta \rightarrow 0$. (This last estimate can be improved to $M(\theta)$ sconst. $\theta^{-1}$, where const. depends only on $A(0,0)$ and $f(0,0)$.)

(II) Let $f=f(x, s, z)$ be a continuous $n$-vector on the $(n+1+m)$-dimensional set $|x|<1,|s|<1,|z|<1$. A necessary and sufficient condition that the initial value problem

$$
x^{\prime}=f(x, s, z) \text { and } x(0)=v,
$$

in which $z$ is a parameter, have a unique solution $x=x(v, s, z)$ which is of class $C^{1}$ in all of its variables for small $|v|,|s|,|z|$ is that there exist an $n$ by $n$ matrix function $A=A(x, s, z)$ and an $n$ by $m$ matrix function $C=C(x, s, z)$ such that $A, C$ are continuous on $|x|<\epsilon,|s|<\epsilon,|z|<\epsilon$ for some $\epsilon>0, A$ is nonsingular, and the $n$ linear differential forms in $d x^{1}, \cdots, d x^{n}, d s, d z^{1}, \cdots, d z^{m}$,

$$
\omega=A d x-b d s+C d z
$$

where $b=A f$, have continuous exterior derivatives on $|x|<\epsilon,|s|<\epsilon,|z|<\epsilon$. 
If $f=\left(f^{1}, \cdots, f^{n}\right), b=\left(b_{1}, \cdots, b_{n}\right), A=\left(a_{j k}\right), C=\left(c_{j \mu}\right)$, then $\omega$ in (7) represents $n$ linear forms $\omega=\left(\omega_{1}, \cdots, \omega_{n}\right)$, where

$$
\omega_{j}=a_{j k} d x^{k}-b_{j} d s+c_{j \mu} d z^{\mu}
$$

$b_{j}=a_{j k} f^{k}, j, k=1, \cdots, n$ and $\mu=1, \cdots, m$.

Let $x=x(v, s, z)$ exist and be of class $C^{1}$ for small $|v|,|s|,|z|$. It is clear from the last part of (6) that the mapping $(v, s, z) \rightarrow(x, s, z)$ is one-to-one if $|v|,|s|,|z|$ are sufficiently small. The first part of (6) shows that this mapping transforms (7) into

$$
\omega=A(\partial x / \partial v) d v+(A(\partial x / \partial z)+C) d z,
$$

where $(\partial x / \partial v)$ and $(\partial x / \partial z)$ denote the Jacobian matrices $\left(\partial x^{i} / \partial v^{k}\right)$ and $\left(\partial x^{i} / \partial z^{\mu}\right)$, where $j, k=1, \cdots, n$ and $\mu=1, \cdots, m$, respectively. Since the forms (7) have continuous exterior derivatives, the forms (9) do also; in particular, the vanishing of the coefficient of $d s$ in (9) implies that the partial derivatives with respect to $s$,

$$
(A(\partial x / \partial v))_{s},(A(\partial x / \partial z)+C)_{s} \text { exist and are continuous; }
$$

cf., e.g., $[9$, p. 351] or the Remark following the proof of (II).

The sufficiency of the conditions in (II) involving (7) when $n=1$ and no parameter $z$ occurs in (6) was proved in [6].

A somewhat more general situation than that in (II) occurs if $x(0)=v$, $x=x(v, s, z)$ are replaced by $x\left(s_{0}\right)=v, x=x\left(v, s, s_{0}, z\right)$. Actually, this case is included in (II) if $f(x, s, z)$ is replaced by $f\left(x, s-s_{0}, z\right)$ and $z$ by the $(m+1)$ vector $\left(s_{0}, z^{1}, \cdots, z^{m}\right)$. Furthermore, (II) is not less general if the parameters $z$ do not occur (for the system (6) can be enlarged to include the relations $z^{\prime}=0, z(0)=z$ ); but the above form of (II) will be needed in Part III.

2. Proof of (I). Let the matrix $A=A(x, s)$ be defined, continuous, nonsingular and such that (4) has a bounded exterior derivative on $|x|<1$, $|s|<1,|z|<1$.

Suppose, if possible, that (2) has two distinct solutions, say $x=x(s)$ and $x=x_{0}(s)$, on an interval $0 \leqq s \leqq \epsilon$ such that $x(\epsilon) \neq x_{0}(\epsilon)$. It can be supposed that

$$
x(s) \neq x_{0}(s) \text { for } 0<s \leqq \epsilon,
$$

for otherwise, the interval $[0, \epsilon]$ can be replaced by a suitable sub-interval $\left[s_{0}, \epsilon\right]$.

Consider a 2-dimensional surface $E=E_{S}$ in the $(x, s)$-space parametrized, for $0 \leqq s \leqq S(\leqq \epsilon)$ and $0 \leqq t \leqq 1$, as follows: $x(s, t)=x(s) t+x_{0}(s)(1-t), s=s$. The boundary $J=J_{S}$ of $E_{S}$ consists of the three $\operatorname{arcs} x=s(s), x=x_{0}(s)$ for $0 \leqq s \leqq S$ and $x=x(S) t+x_{0}(S)(1-t)$ for $0 \leqq t \leqq 1$. The partial derivatives of the $(n+1)$-dimensional vector $(x(s, t), s)$ with respect to $s$ and $t$, respectively, are $\left(x^{\prime} t+x_{0}^{\prime}(1-t), 1\right)$ and $\left(x-x_{0}, 0\right)$. In view of (11) these vectors are linearly 
independent for $0<s<S$ and $0<t<1$. It is also clear that, on $E$,

$$
\begin{aligned}
d x^{j} d x^{k} & =\left\{x^{k \prime}(s, t) \Delta x^{j}-x^{j}(s, t) \Delta x^{k}\right\} d t d s, \\
d x^{i} d s & =\Delta x^{j} d t d s,
\end{aligned}
$$

where $\Delta x^{i}=x^{i}(s)-x_{0}^{j}(s)$.

Let Stokes' formula (1) be applied to $\omega=\omega_{j}$ in (5) and $E=E_{S}, J=J_{S}$. The contribution of the first two arcs of $J$ to the line integral is 0 , by virtue of (2) and (4). Hence, it follows from the existence of a bounded exterior derivative $d \omega_{j}$, from (12) and from Fubini's theorem, that there exists a constant such that

$$
\mid \int_{0}^{1}(A(x(S, t), S) \Delta x(S) d t \mid \leqq \text { Const. } S \max |\Delta x(s)|,
$$

where max refers to $0 \leqq s \leqq S$. Since $x(0)=x_{0}(0)$, it is clear that $A(x(S, t), S)$ $\rightarrow A(0,0)$ as $S \rightarrow 0$ uniformly in $t$. Hence, as $S \rightarrow 0,|A(0,0) \Delta x(S)| \leqq O(S)$ $\cdot \max |\Delta x(s)|$. Since $A$ is nonsingular, there exists a constant $c>0$ such that, as $S \rightarrow 0, c|\Delta x(S)| \leqq o(1) \max |\Delta x(s)|$. This leads to the contradiction $c=0$ if $S$ tends to 0 through a sequence of values such that $|\Delta x(S)|=\max |\Delta x(s)|$ $\neq 0$, and the existence of such a sequence is clear from (11). This proves (I).

3. Proof of (II). Necessity. Suppose that (6) has a unique solution $x=x(v, s, z)$, which is of class $C^{1}$ for small $|v|,|s|,|z|$. Since the Jacobian of $x$ with respect to $v$ is 1 at $s=0$, it follows that the mapping $(v, s, z) \rightarrow(x, s, z)$ has an inverse $v=v(x, s, z), s=s, z=z$ of class $C^{1}$, say, for $|x|<\epsilon,|s|<\epsilon$, $|z|<\epsilon$, with a nonsingular Jacobian matrix $A(x, s, z)=(\partial v / \partial x)=(\partial x / \partial v)^{-1}$. Let $C=C(x, s, z)$ denote the matrix product $-A(\partial x / \partial z)$. Since $d x^{k}=f^{k} d s$ $+\left(\partial x^{k} / \partial v^{j}\right) d v^{j}+\left(\partial x^{k} / \partial z^{\mu}\right) d z^{\mu}$, or $d x=f d s+A^{-1} d v-A^{-1} C d z$, it follows that, with this choice of $A$ and $C, \omega$ in (7) is $\omega=d v$. Hence $\omega$ has the continuous exterior derivative $d \omega=0$.

4. Proof of (II). Sufficiency. Let $A(x, s, z), C(x, s, z)$ be defined and continuous, $A$ nonsingular and (7), where $b=A f$, have a continuous exterior derivative for $|x|<1,|s|<1,|z|<1$.

Let the exterior derivative of (8) be denoted as follows:

$$
d \omega_{j}=\alpha_{j k i} d x^{k} d x^{i}+\beta_{j k} d x^{k} d s+\gamma_{j \mu} d z^{\mu} d s+\delta_{j \mu k} d z^{\mu} d x^{k}+\epsilon_{j \mu \nu} d z^{\mu} d z^{\nu},
$$

where $\alpha_{j k i}=-\alpha_{j i k}, \beta_{j k}, \gamma_{j \mu}, \delta_{j \mu k}, \epsilon_{j \mu \nu}=-\epsilon_{j \mu \mu}$ are continuous functions of $(x, s, z)$.

It follows from Peano's existence theorem and from (I) that $\epsilon>0$ can be chosen so small that $x(v, s, z)$ exists and is continuous for $|v| \leqq \epsilon,|s| \leqq \epsilon$, $|z| \leqq \epsilon$. It will be shown that $x(v, s, z)$ has a continuous partial derivative with respect to the $M$ th component $z^{M}$ of $z, 1 \leqq M \leqq m$, say, at a point $(v, s, z)$.

Let $z=\left(z^{1}, \cdots, z^{m}\right)$ and $z_{0}=\left(z_{0}^{1}, \cdots, z_{0}^{m}\right)$, where $z^{\mu}=z_{0}^{\mu}$ if $\mu \neq M$ and $z^{M} \neq z_{0}^{M}$. Let $x(s)=x(v, s, z)$ and $x_{0}(s)=x\left(v, s, z_{0}\right)$ for $|s| \leqq \epsilon$ (and $v$ and $z^{\mu}$, $\mu \neq M$, are fixed, $\left.|v| \leqq \epsilon,|s| \leqq \epsilon,\left|z_{0}\right| \leqq \epsilon\right)$.

Consider a 2-dimensional surface $E$ in the $(x, s, z)$-space parametrized, 
for $0 \leqq s \leqq \epsilon, 0 \leqq t \leqq 1$, as follows: $x(s, t)=x(s) t+x_{0}(s)(1-t), s=s, z(s, t)$ $=z t+z_{0}(1-t)$. On $E$, the relations (12) and the following relations hold

$$
\begin{aligned}
d z^{\mu} d x^{k} & =0, d z^{\mu} d s=0 \text { if } \mu \neq M \text { and } d z^{\mu} d z^{\nu}=0 \text { if } \mu, \nu=1, \cdots, m, \\
d z^{M} d x^{k} & =x^{k \prime}(s, t) \Delta z^{M} d t d s \text { and } d z^{M} d s=\Delta z^{M} d t d s,
\end{aligned}
$$

if $\Delta z^{M}=z^{M}-z_{0}^{M}$.

Let Stokes' formula (1) be applied to the forms (8) and to the portion of $E$ on which $s$ varies from 0 to $s$ and $t$ from 0 to 1 . If $c_{M}=\left(c_{1 M}, \cdots, c_{n M}\right)$ denotes the column vector which is the $M$ th column of $C=\left(c_{j \mu}\right)$, then the left side of (1) can be written as

$$
\left[\int_{0}^{1} A(x(s, t), s, z(s, t)) d t \Delta x(s)+\int_{0}^{1} c_{M}(x(s, t), s, z(s, t)) d t \Delta z^{M}\right]_{s=0}^{s=s}
$$

where $\Delta x(s)=x(s)-x_{0}(s)$. The right side of (1) is a double integral of the form

$$
\int_{0}^{s} \int_{0}^{1}\left(F(s, t) \Delta x(s)+g(s, t) \Delta z^{M}\right) d t d s,
$$

where $F=F_{M}=\left(f_{j k}\right)$ is a continuous, $n$ by $n$ matrix and $g=g_{M}(s, t)$ $=\left(g_{1 M}, \cdots, g_{n M}\right)$ is a continuous $n$-vector given, respectively, by $f_{j k}$ $=2 \alpha_{j k i} x^{i^{\prime}}(s, t)+\beta_{j k}, g_{j M}=\gamma_{j M}+\delta_{j M k} x^{k^{\prime}}(s, t)$, and the argument of $\alpha, \beta, \gamma, \delta$ is $(x(s, t), s, z(s, t))$.

Let $B=B(s)$ denote the matrix

$$
B(s)=\int_{0}^{1} A(x(s, t), s, z(s, t) d t,
$$

and $u=u(s)$ the vector

$$
u(s)=B(s) \Delta x(s) / \Delta z^{M}+\int_{0}^{1} c_{M}(x(s, t), s, z(s, t)) d t .
$$

Then, by the last part of $(\epsilon)$,

$$
u(0)=\int_{0}^{1} c_{M}(0, v, z(0, t)) d t
$$

and the expression (15) is $[u(s)-u(0)] \Delta z^{M}$. It follows from the equality of (15) and (16) that $u(s)$ has a continuous derivative given by

$$
u^{\prime}=\left(\int_{0}^{1} F d t\right)\left(\Delta x / \Delta z^{M}\right)+\int_{0}^{1} g d t .
$$

If $B(s)$ has an inverse, (17) shows that the last relation can be written as

$$
u^{\prime}=\left(\int_{0}^{1} F d t\right) B^{-1}\left(u-\int_{0}^{1} c_{M} d t\right)+\int^{1} g d t .
$$


As $z_{0} \rightarrow z$, one has $x(s, t) \rightarrow x(s)$ and $z(s, t) \rightarrow z$ uniformly in $(s, t)$. Thus, uniformly in $s, B(s) \rightarrow A(x(s), s, z)$ as $z_{0} \rightarrow z$. In particular, if $\left|z_{0}-z\right|$ is sufficiently small, then $B(s)$ has an inverse and (19) holds. From the expressions above for $F$ and $g=g_{M}$, it is clear that they tend uniformly to continuous limits, (independent of $t$ ), say $F^{0}=F^{0}(v, s, z)$ and $g^{0}=g_{M}^{0}(v, s, z)$, as $z_{0} \rightarrow z$. Thus, as $z_{0} \rightarrow z$, the initial value problem (18), (19) becomes

$$
u^{\prime}=F^{0} A^{-1} u-F^{0} A^{-1} c_{M}+g^{0}, \quad u(0)=c_{M}(v, 0, z),
$$

where the argument of $A^{-1}, c_{M}$ in the differential equation is $(x(s), s, z)$.

Since the linear initial value problem (20) has a unique solution, it follows that (17) tends, as $z_{0} \rightarrow z$, to a limit uniformly in $s$ and that this limit is the solution of (20). This shows that $\partial x(s) / \partial z^{M}=\partial x(v, s, z) / \partial z^{M}$ exists and is continuous; in fact,

$$
u=A(x, s, z) \partial x / \partial z^{M}+c_{M}(x, s, z), \text { where } x=x(v, s, z),
$$

is the solution of $(20)$.

In a similar way, it can be shown that $\partial x / \partial v^{K}$ exists and that

$$
u=A(x, s, z) \partial x / \partial v^{K}, \text { where } x=x(v, s, z),
$$

is the solution of

$$
u^{\prime}=F^{0} A^{-1} u, \quad u(0)=\left(a_{1 K}(v, 0, z), \cdots, a_{n K}(v, 0, z)\right),
$$

cf. [6, Part I], for the case $n=1$. This implies that $\partial x / \partial v^{K}$ is continuous. Hence (II) is proved.

REMARK. The fact that (21) is a solution of (20) and that (22) is a solution of (23) can be abbreviated as

$$
\begin{aligned}
(A(\partial x / \partial z)+C)^{\prime} & =F^{0}(\partial x / \partial z)+G^{0}, \\
(A(\partial x / \partial v))^{\prime} & =F^{0}(\partial x / \partial v),
\end{aligned}
$$

respectively, where $x=x(v, s, z)$, the argument of $A, C, F^{0}, G^{0}$ is $(x, s, z)$ $=(x(v, s, z), s, z), F^{0}$ and $G^{0}$ are the matrices $F^{0}=\left(f_{j k}^{0}\right)=\left(2 \alpha_{j k i} f^{i}+\beta_{j k}\right)$, $G^{0}=\left(g_{j \mu}^{0}\right)=\left(\gamma_{j \mu}+\delta_{j \mu i} f^{i}\right)$, and $\alpha, \beta, \gamma, \delta$ are defined by (13). It follows from (23*) and the Jacobi-Liouville identity that

$$
\operatorname{det}(A(\partial x / \partial v))=[\operatorname{det} A(v, 0, z)] \exp \int_{0}^{s} \operatorname{trace}\left(F^{0} A^{-1}\right) d t,
$$

where the argument of $A(\partial x / \partial v)$ is the same as above and that of $F^{0} A^{-1}$ is $(x, v, z)=(x(v, t, z), v, z)$.

\section{Extremals}

5. It was shown in [4] that, on a 2-dimensional surface $y=y\left(u^{1}, u^{2}\right)$ of class $C^{2}$ embedded in a 3 -dimensional Euclidean $\left(y^{1}, y^{2}, y^{3}\right)$-space, the geodescis are uniquely determined by initial conditions and, in fact, there exist 
local mappings of class $C^{1}$ of $\left(u^{1}, u^{2}\right)$ into geodesic parallel (or polar) coordinates. (The latter mappings need not be of class $C^{2}$; [5].) The proofs in [4] depended on the use of the Gauss-Bonnet formula.

It was pointed out in [8] that the arguments of [4] could be modified to show that if

$$
d s^{2}=g_{j k} d u^{j} d u^{k} \quad\left(g_{j k}=g_{k j}\right)
$$

is a binary, nonsingular, Riemannian metric with coefficients of class $C^{1}$, then a sufficient condition for the local uniqueness of geodesics is that (24) possess a bounded curvature. If, in addition, the curvature is continuous, then the general solution $u=u\left(s, u_{0}, u_{0}^{\prime}\right)$ of the differential equations for geodesics and its derivative $u^{\prime}$ with respect to $s$ are of class $C^{1}$ as functions of $s$ and the initial conditions $u_{0}, u_{0}^{\prime}$ at $s=0$.

The object of Part II is to generalize these theorems in two directions; first, by removing restrictions on the dimensions (of $y$ and of $u$ ) and, second, by replacing the differential equations of geodesics by more general EulerLagrange systems of differential equations.

6. Geodesics. Let (24) be a nonsingular, Riemannian metric with coefficients $g_{j k}=g_{j k}(u)$ of class $C^{1}$ on $|u|<1$, where $u=\left(u^{1}, \cdots, u^{n}\right)$; so that the Christoffel symbols $\Gamma_{j k}^{i}$ of the second kind exist and are continuous. The metric (24) is said to have a continuous [or bounded] curvature tensor $R_{k l l}^{i}$ if the $n^{2}$ Pfaffians

$$
\stackrel{i}{\omega_{k}}=\Gamma_{j k}^{i} d u^{j}
$$

have continuous [or bounded] exterior derivatives; in which case, $R_{k j l}^{i}$ $=-R_{k l j}^{i}$ is defined by

$$
d \omega_{k}^{i}=\left(R_{k j l}^{i}-\Gamma_{k j}^{h} \Gamma_{l h}^{i}+\Gamma_{k l}^{h} \Gamma_{j h}^{i}\right) d u^{j} d u^{l} ;
$$

cf. Weyl [11, pp. 42-44] where $n=2$.

A generalization of the principal results of [8] from 2 to $n$ dimensions is given by

(i) Let (24) be a nonsingular, Riemannian metric with coefficients $g_{i k}=g_{i k}(u)$ of class $C^{1}$ on $|u|<1$. If (24) possesses a bounded curvature tensor, then the initial value problem

$$
u^{i \prime \prime}+\Gamma_{j k}^{i} u^{j \prime} u^{k \prime}=0 \quad \text { and } \quad u(0)=u_{0}, \quad u^{\prime}(0)=u_{0}^{\prime},
$$

where ${ }^{\prime}=d / d$ s and $\left|u_{0}\right|<1$, has a unique solution $u=u\left(s, u_{0}, u_{0}^{\prime}\right)$. If the curvature tensor $R_{j k l}^{3}$ is continuous, then the solution $u=u\left(s, u_{0}, u_{0}^{\prime}\right)$ and its derivative $u^{\prime}\left(s, u_{0}, u_{0}^{\prime}\right)$ are of class $C^{1}$ (in all their variables).

The theorems of [4] concerning geodesics on surfaces can be generalized as follows:

(ii) Let $y=\left(y^{1}, \cdots, y^{m}\right)$ be an $m$-vector, $m \geqq n$, and let $y=y(u)$ be a function of class $C^{2}$ on $|u|<1$ such that the Jacobian matrix $(\partial y / \partial u)$ is of rank $n$. 
Let (24) be the positive definite metric

$$
d s^{2}=|d y|^{2}=\left(y_{j} \cdot y_{k}\right) d u^{j} d u^{k}, \text { where } y_{j}=\partial y / \partial u^{j} .
$$

The corresponding initial value problem (27) for a geodesic of (28) has a unique solution $u=u\left(s, u_{0}, u_{0}^{\prime}\right)$ and $u\left(s, u_{0}, u_{0}^{\prime}\right), u^{\prime}\left(s, u_{0}, u_{0}^{\prime}\right)$ are of class $C^{1}$.

7. Proofs of (i) and (ii). Although (i) and (ii) will be generalized further below, it seems worthwhile to give direct, short proofs for them.

Ad (i). Let $\left(g^{j k}\right)$ denote the matrix inverse to the matrix $\left(g_{j k}\right)$. The Hamiltonian form for the system in (27) is

$$
u^{i \prime}=g^{i j} v_{j}, \quad v_{i}^{\prime}=-\frac{1}{2}\left(\partial g^{j k} / \partial u^{i}\right) v_{j} v_{k},
$$

where the first part of (29) serves to define $v=\left(v_{1}, \cdots, v_{n}\right)$. It is readily verified that (29) is equivalent to

$$
u^{j \prime}-g^{j k} v_{k}=0, \quad v_{j}^{\prime}-v_{i} \Gamma_{j k}^{i} u^{k \prime}=0 .
$$

Consider the $2 n$ linear forms in the $2 n+1$ variables $\left(u^{1}, \cdots, u^{n}, v_{1}, \cdots\right.$, $\left.v_{n}, s\right)$,

$$
d u^{j}-g^{j k} v_{k} d s, \quad d v_{j}-v_{i} \Gamma_{j k}^{i} d u^{k} .
$$

These forms belong to the system (29) in the same way as (4) belongs to (2); for, in matrix-vector notation, (31) can be written as $A(d u, d v)-b d s$, where $A$ is the $2 n$ by $2 n$ nonsingular matrix

$$
A=\left(\begin{array}{cc}
I & 0 \\
-v_{i} \Gamma_{j k}^{i} & I
\end{array}\right)
$$

$b$ is the $2 n$-vector $\left(g^{1 i_{j}}, \cdots, g^{n i} v_{j}, 0, \cdots, 0\right), I$ is the unit $n$ by $n$ matrix, and $A(d u, d v)$ is the product of $A$ and the $2 n$-vector $(d u, d v)$. In view of the definition of the curvature tensor in terms of (25), it is clear that (I) and (II) imply (i).

Ad (ii). The assertion (ii) follows from (i) if it is verified that (28) has a continuous curvature tensor. Since $\Gamma_{j k}^{l}=g^{l i}\left(y_{j k} \cdot y_{l}\right)$, where $y_{j k}=\partial^{2} y / \partial u^{i} \partial u^{k}$, it follows that

$$
\Gamma_{j k}^{i} d u^{k}=g^{i l}\left(y_{l} \cdot d y_{j}\right) .
$$

The forms $d y_{j}$ have continuous exterior derivatives, $d\left(d y_{j}\right)=0$, while the factors $g^{i l} y_{l}$ are of class $C^{1}$. Hence (32) has continuous exterior derivatives; cf., e.g., $[9$, p. 350]. This proves (ii).

8. Extremals. Assertion (i) will be generalized so as to become applicable to problems of extremals for more general parametric problems of the calculus of variations. The following set of conditions on a Lagrangian $F$ will be re- 
ferred to as condition $(\dagger)$.

(†) Let $F\left(u, u^{\prime}\right)$ be defined for $u$ near a given point $u=u_{0}$, and for all $u^{\prime}$, where $u, u^{\prime}$ are $n$-vectors. Suppose that $F$ and $F^{j}=\partial F / \partial u^{j^{\prime}}, j=1, \cdots, n$, are of class $C^{1}$ as functions of $\left(u, u^{\prime}\right)$. Suppose also that $F$ is positively homogeneous of degree 1 in the variable $u^{\prime}$ (that is, that $F\left(u, c u^{\prime}\right)=c F\left(u, u^{\prime}\right)$, where $c>0)$ and that, for $\left(u, u^{\prime}\right)$ in a vicinity of a given point $\left(u, u^{\prime}\right)=\left(u_{0}, u_{0}^{\prime}\right)$,

$$
\operatorname{det}\left(\begin{array}{ll}
F^{i j} & u^{i \prime} \\
u^{i^{\prime}} & 0
\end{array}\right) \neq 0 .
$$

In (33) and below, the following abbreviations are used for partial derivatives of $F$,

$$
F_{j}=\partial F / \partial u^{j}, \quad F^{j}=\partial F / \partial u^{j \prime}, \quad F^{i j}=\partial^{2} F / \partial u^{i \prime} \partial u^{j \prime}, \quad F_{i}^{j}=\partial^{2} F / \partial u^{i} \partial u^{j \prime} .
$$

Also, $F_{u}$ denotes the vector $\left(F_{1}, \cdots, F_{n}\right)$ and $F^{u^{\prime}}$ the vector $\left(F^{1}, \cdots, F^{n}\right)$.

Under condition $(\dagger)$ on $F$, there exist functions $H(u, v)$, called Hamiltonian functions of $F$, defined for $(u, v)=\left(u^{1}, \cdots, u^{n}, v_{1}, \cdots, v_{n}\right)$ near $\left(u_{0}, F^{u^{\prime}}\left(u_{0}, u_{0}^{\prime}\right)\right)$ with the properties that $H$ and $H^{j}=\partial H / \partial v_{j}, j=1, \cdots, n$, are of class $C^{1}$ as functions of $(u, v)$; the vector $H^{v}=\left(H^{1}, \cdots, H^{n}\right)$ does not vanish; $H\left(u, F^{u^{\prime}}\left(u, u^{\prime}\right)\right) \equiv 0$ for $\left(u, u^{\prime}\right)$ near $\left(u_{0}, u_{0}^{\prime}\right)$; there exists a function $\lambda=\lambda\left(u, u^{\prime}\right)>0$ of class $C^{1}$ such that

$$
v=F^{u^{\prime}}\left(u, u^{\prime}\right), \quad u^{\prime}=\lambda H^{v}(u, v)
$$

are reciprocal relations; finally, for $\left(u, u^{\prime}\right)$ near $\left(u_{0}, u_{0}^{\prime}\right)$, the Euler-Lagrange system of differential equations (of second order for $u$ ),

$$
\left(F^{u^{\prime}}\right)^{\prime}-F_{u}=0 \text {, }
$$

is equivalent to the Hamiltonian system (of first order for $(u, v)$ ),

$$
u^{\prime}=\lambda H^{v}(u, v), \quad v^{\prime}=-\lambda H_{u}(u, v),
$$

where $H_{u}$ is the vector $\left(H_{1}, \cdots, H_{n}\right)$ and $H_{j}=\partial H / \partial u^{i}$. See [2, pp. 216-218], where $F$ is assumed to be of class $C^{2}$.

The generalization of (i) to be verified below is

$(\alpha)$ Let $F\left(u, u^{\prime}\right)$ satisfy conditions $(\dagger)$, let $H(u, v)$ be a Hamiltonian function (and $\lambda\left(u, u^{\prime}\right)>0$ a multiplier for which (34) holds). If the $n$ Pfaffians in the variables $(u, v)$,

$$
\omega_{i}=F_{i}^{j}\left(u, H^{v}(u, v)\right) d u^{j}, \text { where } i=1, \cdots, n,
$$

have bounded exterior derivatives, then the initial value problem belonging to (35) and $u(0)=u_{*}, u^{\prime}(0)=u_{*}^{\prime}$ has a unique solution $u=u\left(s, u_{*}, u_{*}^{\prime}\right)$ for $\left(u_{*}, u_{*}^{\prime}\right)$ near $\left(u_{0}, u_{0}^{\prime}\right)$. If the forms (37) have continuous exterior derivatives, then $u\left(s, u_{*}, u_{*}^{\prime}\right)$ and $u^{\prime}\left(s, u_{*}, u_{*}{ }^{\prime}\right)$ are of class $C^{1}$ (as functions of their $2 n+1$ variables). 
9. Proof of $(\alpha)$. The relations between the Lagrangian $F$ and a Hamiltonian $H$ and multiplier $\lambda$ imply that, by virtue of $(34), \lambda\left(u, u^{\prime}\right) H_{u}(u, v)$ $=-F_{u}\left(u, u^{\prime}\right)$; cf. [2, p. 222]. Hence, by Euler's relation for homogeneous functions, $\lambda H_{i}=-F_{i}^{j} u^{j^{\prime}}$. Thus, the Hamiltonian system (36) can be written as $u^{i \prime}=\lambda\left(u, u^{\prime}\right) H^{i}(u, v), v_{i}^{\prime}=-F_{i}^{j}\left(u, \lambda H_{v}\right) u^{j^{\prime}}$, that is, as the vector equation $A\left(u^{\prime}, v^{\prime}\right)-b=0$, where $A$ is the $2 n$ by $2 n$ nonsingular matrix

$$
A=\left(\begin{array}{cc}
I & 0 \\
-F_{i}^{j} & I
\end{array}\right)
$$

and $b$ is the $2 n$-vector $\left(\lambda H^{v}, 0\right)$.

Thus the two assertions of $(\alpha)$ follow from (I), (II), respectively, if the $2 n$ forms in $d u^{1}, \cdots, d u^{n}, d v_{2}, \cdots, d v_{n}, d s$,

$$
d u^{i}-\lambda H^{i} d s, \quad d v_{i}+F_{i}^{j}\left(u, \lambda H_{v}\right) d u^{j}
$$

have bounded or continuous exterior derivatives. The first $n$ forms are of class $C^{1}$ and need not be considered. The question of the differentiability of the second $n$ forms reduces to the question of the differentiability of (37), for the homogeneity of $F$ and $\lambda>0$ imply that $F_{i}^{\prime}\left(u, \lambda H^{v}\right)=F_{i}^{j}\left(u, H^{v}\right)$. Thus $(\alpha)$ is proved.

10. Generalized geodesics. The following set of conditions on functions $F$ and $G$ will be referred to as condition $(\dagger \dagger)$.

$(\dagger \dagger)$ Let $y, y^{\prime}$ be $m$-vectors. Let $G\left(y, y^{\prime}\right)$ be defined for $y$ near a point $y=y_{0}$ and for all $y^{\prime}$ and have the properties that $G$ is of class $C^{2}$, is positively homogeneous of degree 1 in $y^{\prime}$, and that

$$
\operatorname{det}\left(\begin{array}{ll}
G^{\mu \nu} & y^{\mu^{\prime}} \\
y^{\nu \prime} & 0
\end{array}\right) \neq 0 \text { for }\left(y, y^{\prime}\right) \text { near }\left(y_{0}, y_{0}^{\prime}\right) \text {. }
$$

Let $y=y(u)$ be a $C^{2}$ function of an $n$-vector $u$ near $u=0$, where $m \geqq n$, such that the rank of the Jacobian matrix $(\partial y / \partial u)$ is $n$, that $y(0)=y_{0}$ and that there exists an $n$-vector $u_{0}^{\prime}$ satisfying $y_{0}^{\prime}=(\partial y(0) / \partial u) u_{0}^{\prime}$. Put

$$
F\left(u, u^{\prime}\right)=G\left(y(u),(\partial y(u) / \partial u) u^{\prime}\right) .
$$

Finally, let $(33)$ hold for $\left(u, u^{\prime}\right)=\left(0, u_{0}^{\prime}\right)$.

The notation in (38) is analogous to that in (33). As in $\$ 6, y_{j}$ will denote the $m$-vector $\partial y / \partial u^{i}$; the $\mu$ th component of this vector will be written $y_{j}^{\mu}$. The $m$-vector $(\partial y / \partial u) u^{\prime}$ has the $\mu$ th component $y_{j}^{\mu} u u^{j^{\prime}}$. A dot denotes scalar multiplication of $m$-vectors.

The calculus of variations problem belonging to the Lagrangian $F$ is merely the problem belonging to $G$ induced on the $n$-dimensional manifold $y=y(u)$. Solutions of the corresponding Euler-Lagrange equations (35) are called generalized geodesics; cf. [2, pp. 283-284]. A generalization of (ii) is the following:

( $\beta$ ) Under condition $(\dagger \dagger)$, a generalized geodesic $u=u(s)=u\left(s, u_{*}, u_{*}^{\prime}\right)$ is 
uniquely determined by its initial conditions $\left(u(0), u^{\prime}(0)\right)=\left(u_{*}, u_{*}^{\prime}\right)$ near $\left(u_{0}, u_{0}^{\prime}\right)$; furthermore, $u\left(s, u_{*}, u_{*}^{\prime}\right), u^{\prime}\left(s, u_{*}, u_{*}^{\prime}\right)$ are of class $C^{1}$ (as functions of their $2 n+1$ arguments).

11. Proof of $(\beta)$. Let $K(y, z)$ be a Hamiltonian function belonging to $G\left(y, y^{\prime}\right)$ and $\lambda=\lambda\left(y, y^{\prime}\right)>0$ a corresponding multiplier. It can be supposed that $K$ is of class $C^{2}$ and that $\lambda$ is of class $C^{1}$. The equations

$$
v_{i}=z \cdot y_{i}(u), \quad y_{j}(u) u^{j^{\prime}}=\lambda K^{2}(y(u), z)
$$

can be solved for the $m$-vector $z$ and for $u^{\prime} / \lambda$ as functions of $u$ and $v$ $=\left(v_{1}, \cdots, v_{n}\right)$, for $(u, v)$ near the point $\left(u_{0}, v_{0}\right)$ with coordinates $u_{0}^{j}=0$ and $v_{i 0}=G^{y^{\prime}}\left(y_{0}, y_{0}^{\prime}\right) \cdot y_{i}(0)$, say $z=z(u, v)$ and $u^{\prime}=\lambda \phi(u, v)$, where $z, \phi$ are of class $C^{1}$. A Hamiltonian function $H(u, v)$ belonging to $F\left(u, u^{\prime}\right)$ is given by $H(u, v)$ $=K(y(u), z(u, v))$; the corresponding multiplier is $\lambda\left(y(u),(\partial y / \partial u) u^{\prime}\right)$.

These facts are proved in [2, pp. 284-286], where it is assumed that $y=y(u)$ is of class $C^{3}$ and where assumptions (33), (38) are replaced by the stronger condition that the matrix $\left(G^{\mu \nu}\left(y_{0}, y_{0}^{\prime}\right)\right)$ is positive definite on the linear $(n-1)$-space orthogonal to $y_{0}^{\prime}$. It will be verified that these facts remain valid under the present conditions.

The equations (40) can be solved for $z$ and $u^{\prime} / \lambda$ as functions of $(u, v)$ if the $n+m$ linear equations in the $n+m$ variables $(\rho, \sigma)=\left(\rho_{1}, \cdots, \rho_{m}, \sigma^{1}, \cdots\right.$, $\left.\sigma^{n}\right)$,

$$
K^{\mu \nu} \rho_{\nu}+y^{\mu}{ }_{j} \sigma^{j}=0, \quad y^{\nu}{ }_{i} \rho_{\nu}=0,
$$

where $\mu=1, \cdots, m$ and $i=1, \cdots, n$ and the argument of $y_{j}^{\mu}$ is $u=0$ and that of $K^{\mu \nu}$ is $(y, z)=\left(y_{0}, z_{0}\right)=\left(y_{0}, G^{y^{\prime}}\left(y_{0}, y_{0}^{\prime}\right)\right)$, have no solution $(\rho, \sigma) \neq(0,0)$.

Let $(\rho, \sigma)$ be a solution. Note that

$$
0=u_{0}^{i \prime} y_{i}^{\nu} \rho_{\nu}=y_{0}^{\nu \prime} \rho_{\nu}
$$

and that

$$
G^{\kappa \mu} K^{\mu \nu} \rho_{\nu}+G^{\kappa \mu} y_{j}^{\mu} \sigma^{j}=0 .
$$

Differentiating $y^{\nu^{\prime}}=\lambda K^{\nu}\left(y, G^{y^{\prime}}\left(y, y^{\prime}\right)\right)$, the analogue of the last relation of (34), with respect to $y^{{ }^{\prime \prime}}$ gives the fundamental relation

$$
\delta^{\kappa \nu}=\lambda K^{\nu \mu} G^{\mu \kappa}+\left(\partial \lambda / \partial y^{\kappa^{\prime}}\right) K^{\nu}
$$

where $\delta^{k \nu}$ is 1 or 0 according as $\kappa=\nu$ or $\kappa \neq \nu ;$ [2, p. 218]. The last three relations imply

$$
\rho_{\nu}+\lambda G^{\nu \mu} y_{j}^{\mu} \sigma^{j}=0
$$

since $K^{\nu}=y^{\nu} / \lambda$. Multiplying this last equation by $y_{m}^{\nu}$ gives, by (39), $y_{m}^{\nu} \rho_{\nu}$ $+\lambda F^{j m} \sigma^{j}=0$, that is, $F^{j m} \sigma^{j}=0$ for $m=1, \cdots, n$. The condition (33) implies, therefore, that the $n$-vector $\sigma$ is a multiple of $u_{0}^{\prime}$, say $\sigma=c u_{0}^{\prime}$. But $G^{\nu \mu} y_{j}^{\mu} \sigma^{j}$ $=c G^{\nu \mu} y_{0}^{\mu \prime}=0$, where the last equality follows from the homogeneity of $G$. 
Consequently, the last formula line gives $\rho=0$. Hence, by the first set of linear equations in $\rho$ and $\sigma, 0=y_{j}^{\mu} \sigma^{j}=c y_{0}^{\mu \prime}$ and so $c=0$, that is, $\sigma=0$.

Consequently, the solutions of $(40), z=z(u, v)$ and $u^{\prime} / \lambda=\phi(u, v)$, exist and are of class $C^{1}$. Hence $H(u, v)=K(y(u), z(u, v))$ is of class $C^{1}$. Also, by (40),

$$
\lambda H^{i}=\lambda K^{\nu}\left(\partial z^{\nu} / \partial v_{i}\right)=y_{j}^{\nu} u^{j}\left(\partial z^{\nu} / \partial v_{i}\right) .
$$

Differentiating the first equation in (40) with respect to $v_{k}$, where $z=z(u, v)$, gives $\delta^{i k}=y_{i}^{\nu}\left(\partial z^{\nu} / \partial v_{k}\right)$. Hence the last formula line reduces to $\lambda H^{i}=u^{i \prime}$, that is, the last equation in (34). In particular, $H^{i}=u^{i} / \lambda=\phi(u, v)$ is of class $C^{1}$ and the vector $H^{v}=\left(H^{1}, \cdots, H^{n}\right)$ is not 0 .

In order to verify $H\left(u, F^{u^{\prime}}\left(u, u^{\prime}\right)\right) \equiv 0$, that is, $K\left(y(u), z\left(u, F^{u^{\prime}}\left(u, u^{\prime}\right)\right)\right) \equiv 0$, it is sufficient to show that $z^{\mu}\left(u, F^{u^{\prime}}\right)=G^{\mu}\left(y, y^{\prime}\right)$, where $y^{\nu}=y^{\nu}(u)$ and $y^{\nu^{\prime}}$ $=y_{k}^{\nu}(u) u^{k^{\prime}}$. If $v_{i}$ is replaced by $F^{i}=G^{\mu} y_{i}^{\mu}$, the first part of $(40)$ gives $\left(G^{\mu}-z^{\mu}\right) y_{i}^{\mu}$ $=0$ for $i=1, \cdots, n$. Since the Jacobian matrix $(\partial y / \partial u)$ is of rank $m, G^{\mu}-z^{\mu}$ $=0$.

From (33) and the fact that $H, H^{v} \neq 0$ are of class $C^{1}$, that $H\left(u, F^{u^{\prime}}\right) \equiv 0$, that the last part of (34) holds follows that the first part of (34) holds and that (35) and (36) are equivalent; cf., e.g., [2, pp. 218-222]. In other words, $H$ is a Hamiltonian function of $F$ and $\lambda$ is the corresponding multiplier.

In view of $H=K(y(u), z(u, v))$, the last set of equations in (36) can be written as

$$
v_{i}^{\prime}=-\lambda\left(K_{\nu} \cdot y_{i}+K^{z} \cdot z_{i}\right)
$$

where $z_{i}$ is the vector $\partial z(u, v) / \partial u^{i}$ and the argument of $\lambda$ and the derivatives of $K$ are $\left(y(u),(\partial y / \partial u) u^{\prime}\right)$ and $(y(u), z(u, v))$, respectively.

The first set of equations in (40) shows that $y_{j} \cdot z_{i}=-z \cdot y_{i j}$. This relation and the last part of (40) show that the last set of differential equations can be written as

$$
v_{i}^{\prime}=-\lambda K_{y} \cdot y_{i}-z \cdot y_{i j} u^{j^{\prime}} .
$$

Consequently, a solution of (36) is a solution of the system consisting of the first part of (36), $u^{\prime}-\lambda H^{v}=0$, and of (41); and conversely. This system can be written as $A\left(u^{\prime}, v^{\prime}\right)-b=0$, where $A$ is the nonsingular, $2 n$ by $2 n$ matrix

$$
A=\left(\begin{array}{cc}
I & 0 \\
z \cdot y_{i j} & I
\end{array}\right)
$$

$b$ is the $2 n$-vector $\lambda\left(H^{1}, \cdots, H^{n},-K_{y} \cdot y_{1}, \cdots,-K_{y} \cdot y_{n}\right)$ and $z=z(u, v)$, $y=y(u)$.

Correspondingly, (II) suggests the consideration of the $2 n$ Pfaffiians in $d u^{1}, \cdots, d u^{n}, d v_{1}, \cdots, d v_{n}, d s$,

$$
d u^{i}-\lambda H^{i} d s, \quad d v_{i}+\lambda\left(K_{y} \cdot y_{i}\right) d s+\left(z \cdot y_{i j}\right) d u^{j} .
$$


The first $n$ of these forms and the $n$ forms $d v_{i}+\lambda\left(K_{y} \cdot y_{i}\right) d s$ are of class $C^{1}$ and, therefore, have continuous exterior derivatives. The forms $\left(z \cdot y_{i j}\right) d u^{i}=z \cdot d y_{i}$ have continuous exterior derivatives, since $d\left(d y_{i}\right)=0$ and $z=z(u, v)$ is of class $C^{1}$; cf., e.g., [9, p. 350]. Thus $(\beta)$ follows from (II).

\section{A THEOREM OF Frobenius}

12. The object of Part III is to prove the following:

$\left(^{*}\right)$ Let $\omega_{1}, \cdots, \omega_{m}$ denote $m$ linearly independent linear forms in $d x^{1}, \cdots, d x^{n}$ with continuous coefficients in $|x|<1$ possessing continuous exterior derivatives $d \omega_{1}, \cdots, d \omega_{m}$ and satisfying the integrability conditions

$$
\left(\omega_{1} \cdots \omega_{m}\right) d \omega_{\mu} \equiv 0 \text { for } \mu=1, \cdots, m .
$$

Then, in a vicinity of $y=0$, there exists a mapping $x=x(y)$ (that is, $x^{j}$ $=x^{j}\left(y^{1}, \cdots, y^{n}\right)$ for $\left.j=1, \cdots, n\right)$ satisfying $x(0)=0$, of class $C^{1}$, with nonvanishing Jacobian, det $\left(\partial x^{j} / \partial y^{k}\right) \neq 0$, and transforming $\omega_{1}, \cdots, \omega_{m}$ into linear forms in $d y^{1}, \cdots, d y^{m}$ (with coefficients which are continuous functions of $\left.y^{1}, \ldots, y^{n}\right)$.

$\left(^{*}\right)$ is a theorem of Frobenius and is known if the $\omega_{1}, \cdots, \omega_{m}$ are of class $C^{1}$; cf., e.g., [1, pp. 99-100]. Under the condition that $\omega_{1}, \cdots, \omega_{m}$ are continuous and possess continuous exterior derivatives, the integrability conditions (42) are necessary, as well as sufficient, for the assertion of $\left(^{*}\right)$. Clearly, $(*)$ implies the following (cf. $\$ 13$ below):

REMARK. Let $\omega_{1}, \cdots, \omega_{m}$ denote $m$ linearly independent linear forms in $d x^{1}, \cdots, d x^{n}$ with continuous coefficients on $|x|<1$. A necessary and sufficient condition for the existence of a mapping $x=x(y)$ of class $C^{1}$ for small $|y|$, having a nonvanishing Jacobian, satisfying $x(0)=0$, and transforming $\omega_{1}, \cdots, \omega_{m}$ into forms in $d y^{1}, \cdots, d y^{m}$ is that there exist a continuous, nonsingular, $m$ by $m$ matrix $\left(e^{\nu \mu}(x)\right)$ for small $|x|$ such that the forms $\omega_{\nu 0}=e^{\nu \mu} w_{\mu}$ have continuous exterior derivatives $d w_{\nu 0}$ satisfying the integrability conditions $\left(\omega_{10} \omega_{20} \cdots \omega_{m 0}\right) d \omega_{\mu 0}=0$ for $\mu=1, \cdots, m$.

Let $p_{\mu k}(x)$, where $\mu=1, \cdots, m$ and $k=1, \cdots, n$, denote the coefficients in $\omega_{\mu}$,

$$
\omega_{\mu}=p_{\mu k}(x) d x^{k}, \quad \quad \mu=1, \cdots, m .
$$

The forms (43) are said to be linearly independent if the rectangular matrix $\left(p_{\mu k}(x)\right)$ is of rank $m$ (at every point $\left.x\right)$; so that $m \leqq n$. The product in (42) is the formal product under the associative law and the exterior multiplication rule, $d x^{j} d x^{k}=-d x^{k} d x^{j}$. A form is said to be identically 0 when its coefficients are identically 0 . The condition (42) means that if, at a fixed $x$, the (independent) variables $d x^{1}, \cdots, d x^{n}$ are chosen so as to satisfy $\omega_{1}=\cdots$ $=\omega_{m}=0$, then the (exterior) quadratic forms $d \omega_{1}, \cdots, d \omega_{m}$ vanish. The assertion of $\left({ }^{*}\right)$ means that $x=x(y)$ transforms (43) into the form 


$$
\sum_{\nu=1}^{m} q_{\mu \nu}(y) d y^{\nu}
$$

where $\left(q_{\mu \nu}(y)\right)$ is a nonsingular $m$ by $m$ matrix in a vicinity of $y=0$. It is clear, from the definition of exterior derivative, that if (43) has a continuous exterior derivative, then its image (44) under a $C^{1}$-mapping $x=x(y)$ has a continuous exterior derivative. In particular, the partial derivatives $\partial q_{\mu \nu} / \partial y^{i}$ exist and are continuous for $i=m+1, \cdots, n$; cf. (vii) in $[9$, p. 351], or the proof of $(*)$ below.

If $m=1$ and $n=2$, then $\left({ }^{*}\right)$ reduces to the case $n=1$ of (II) (in this case, the integrability condition (42) is trivially satisfied). If $m=1$ and $n=3$, let $\omega=p_{1} d x^{1}+p_{2} d x^{2}+p_{3} d x^{3}$, where $\omega$ is continuous, satisfies $p_{1}^{2}+p_{2}^{2}+p_{3}^{2} \neq 0$ and has a continuous exterior derivative $d \omega=r_{1} d x^{2} d x^{3}+r_{2} d x^{3} d x^{1}+r_{3} d x^{1} d x^{2}$ (so that $r_{1}=p_{3 y}-p_{2 z}, r_{2}=p_{1 z}-p_{3 x}, r_{3}=p_{2 x}-p_{1 y}$ if $p_{1}, p_{2}, p_{3}$ are of class $\left.C^{1}\right)$. The integrability condition (42) in this case means that $p_{1} r_{1}+p_{2} r_{2}+p_{3} r_{3} \equiv 0$. In this case, $\left({ }^{*}\right)$ asserts that there exists a mapping $x \rightarrow y$ of class $C^{1}$ (in a vicinity of $x=0$ ), with nonvanishing Jacobian, transforming $\omega$ into the form $q\left(y^{1}, y^{2}, y^{3}\right) d y^{1}$. In other words, $\omega$ possesses a nontrivial integrating factor $1 / q$ in a sufficiently small vicinity of $x=0$.

13. Note that if $\left(e_{\mu \nu}(x)\right)$ is a continuous, nonsingular, $m$ by $m$ matrix, then the problem of reducing (43) to the form (44) is equivalent to reducing the forms $\omega_{10}, \cdots, \omega_{m 0}$ to the form (44), where

$$
\omega_{\mu}=\sum_{v=1}^{m} e_{\mu \nu} \omega_{\nu 0}
$$

If the functions $e_{\mu \nu}$ are of class $C^{1}$, then $\omega_{10}, \cdots, \omega_{m 0}$ satisfy the conditions of $\left.{ }^{*}\right)$ if and only if $\omega_{1}, \cdots, \omega_{m}$ do. If the functions $e_{\mu \nu}$ are only continuous, however, then $\omega_{10}, \cdots, \omega_{m 0}$ need not possess exterior derivatives when $\omega_{1}, \cdots, \omega_{m}$ do.

Let $m<n$ and consider a system of $n-m$ linearly independent, homogeneous, linear partial differential equations

$$
X_{\tau}(f) \equiv \sum_{k=1}^{n} a_{\tau k}(x) \partial f / \partial x^{k}=0 \quad(\tau=1, \cdots, n-m)
$$

with continuous coefficients $a_{\tau k}(x)$. This system is said to be complete on a given domain if there exist $m$ solutions $f=y^{\mu}\left(x^{1}, \cdots, x^{n}\right)$, where $\mu=1, \cdots$, $m$, such that the $m$ by $n$ matrix $\left(\partial y^{\mu} / \partial x^{k}\right)$ is of rank $m$. When the coefficients $a_{\tau k}$ in (46) are of class $C^{1}$, necessary and sufficient conditions for the (local) completeness of (46) can be expressed in terms of the $a_{\tau k}$ and their partial derivatives. For example, after taking suitable linear conditions of (46) and re-numbering the independent variables, it can be supposed that (46) is of the form 


$$
X_{\tau}(f) \equiv \partial f / \partial x^{\tau}+\sum_{\mu=1}^{m} a_{\tau \mu}(x) \partial f / \partial x^{\mu}, \quad \text { where } \quad \tau=m+1, \cdots, n .
$$

In which case, the criterion for (local) completeness is $X_{\sigma}\left(a_{\tau \mu}\right)-X_{\tau}\left(a_{\sigma \mu}\right)=0$, where $\mu=1, \cdots, m ; \sigma, \tau=m+1, \cdots, n$; e.g., Ostrowski [10], where references are given to Gillis, Schmidt and Perron, and [1, pp. 103-105]. On the other hand, the problem of finding solutions $f=y^{1}, \cdots, y^{m}$ of $(47)$ of the type specified is equivalent to reducing the forms $\omega_{10}, \cdots, \omega_{m 0}$, where

$$
\omega_{\mu 0}=d x^{\mu}-\sum_{\tau=m+1}^{n} a_{\tau \mu}(x) d x^{\tau} \text { and } \mu=1, \cdots, m,
$$

into the form (44), in the manner specified in $\left({ }^{*}\right)$; cf., e.g., [1, pp. 103-105]. It follows from $(*)$ that a sufficient condition for the (local) completeness of $(47)$ is that there exists a continuous, nonsingular, $m$ by $m$ matrix $\left(e_{\mu \nu}\right)$ such that (45) satisfies the conditions of $\left({ }^{*}\right)$. This formulation of a sufficient condition avoids the assumption that the coefficients $a_{\tau \mu}$ in (48) are of class $C^{1}$.

If the forms $\omega_{1}, \cdots, \omega_{m}$ in $\left(^{*}\right)$ are in the normal form (48), the existence of continuous exterior derivatives implies that the $a_{\tau \mu}$ have continuous partial derivatives with respect to $x^{1}, \cdots, x^{m}$ (but not necessarily with respect to $\left.x^{m+1}, \cdots, x^{n}\right)$. In this case, the proof of $(*)$ shows that $\left({ }^{*}\right)$ is equivalent to a theorem ([3]; cf. also $\left[7\right.$, p. 766]) on the Jacobi system $\omega_{10}=\cdots=\omega_{m 0}=0$; that is, in the assertion of $\left(^{*}\right), x=x(y)$ can be chosen so that $x^{\tau} \equiv y^{\tau}$ if $\tau$ $=m+1, \cdots, n$. In fact, the proof of $\left({ }^{*}\right)$ will show that this can always be realized when the coefficients of (43) are such that

$$
\operatorname{det}\left(p_{\mu \nu}\right) \neq 0 \text {, where } \mu, \nu=1, \cdots, m \text {. }
$$

This gives another proof, without successive approximations, of the theorems of [3]; cf. also Theorems (II) and (III) of [7, p. 760 and p. 766].

14. The proof of $\left({ }^{*}\right)$ will depend on (II) and on the following:

Lemma. Let $x, s, z$ be as in (II); $A=A(x, s, z)$ a continuous, nonsingular, $n$ by $n$ matrix, $C=C(x, s, z)$ a continuous $m$ by $n$ matrix, $b=b(x, s, z)$ a continuous $n$-vector on $|x|<1,|s|<1,|z|<1$ such that the $n$ Pfaffians (7), that is, (8) have continuous exterior derivatives. In addition, let the forms (8) satisfy the integrability condition $\left(\omega_{1} \omega_{2} \cdots \omega_{n}\right) d \omega_{k}=0$ for $k=1, \cdots, n$. Let $x=x(v, s, z)$ be the solution of (6), where $f=A^{-1} b$, and let $\epsilon>0$ be so small that $\operatorname{det}(\partial x / \partial v) \neq 0$ for $|x|<\epsilon,|s|<\epsilon,|z|<\epsilon$. Then the matrix $(A(\partial x / \partial v))^{-1}(A(\partial x / \partial z)+C)$ is independent of $s$.

Proof. The mapping $(x, s, z) \rightarrow(v, s, z)$ transforms (7) into (9), where (10) holds. The terms involving $d v d s$ and $d z^{M} d s$ in $d \omega$ are $-(A(\partial x / \partial v))_{s} d v d s$ $-\left(A\left(\partial x / \partial z^{M}\right)+c_{M}\right)_{s} d z^{M} d s$, where the subscript $s$ denotes partial differentiation. At a given point $(v, s, z)$, determine the (independent) variables $d v, d s$, $d z$ by the requirement that 


$$
d v / d z^{M}=-(A(\partial x / \partial v))^{-1}\left(A\left(\partial x / \partial z^{M}\right)+c_{M}\right),
$$

$d z^{\mu}=0$ if $\mu \neq M$ and $d s$ is arbitrary. Then $\omega=0$, by (9). Hence the integrability conditions imply that $d \omega=0$. But $d \omega$ reduces to

$$
\left\{(A(\partial x / \partial v))_{s}(A(d x / \partial v))^{-1}\left(A\left(\partial x / \partial z^{M}\right)+c_{M}\right)-\left(A\left(\partial x / \partial z^{M}\right)+c_{M}\right)_{s}\right\} d z^{M} d s ;
$$

so that this coefficient $\{\cdots\}$ of $d z^{M} d s$ is 0 .

On the other hand, (21) is a solution of (20) and (22) is a solution of (23). If $(A(\partial x / \partial v))_{s}$ is replaced by $F^{0} A^{-1}(A(\partial x / \partial v))=F^{0}(\partial x / \partial v)$ and $\left(A\left(\partial x / \partial z^{M}\right)+c_{M}\right)_{s}$ by $F^{0} A^{-1}\left(A\left(\partial x / \partial z^{M}\right)+c_{M}\right)-F^{0} A^{-1} c_{M}+g^{0}=F^{0}\left(\partial x / \partial z^{M}\right)+g^{0}$, the equation $\{\cdots\}=0$ shows that $F^{0} A^{-1} c_{M}-g^{0} \equiv 0$. Consequently, (20) reduces to $u^{\prime}=F^{0} A^{-1} u, u(0)=c_{M}(v, 0, z)$. In other words, (21) and (22) are solutions of the same linear, homogeneous system of ordinary differential equations.

Since $A(\partial x / \partial v)$ is a fundamental matrix for this system (that is, the columns of $A(\partial x / \partial v)$ are linearly independent solution vectors of this system), it follows, from the superposition principle, that if $u=u(s)$ is any solution, then the vector $(A(\partial x / \partial v))^{-1} u(s)$ is independent of $s$. This proves the lemma.

15. Proof of $\left({ }^{*}\right)$. Let the forms $(43)$ be those occurring in $\left({ }^{*}\right)$. After a suitable re-numbering of the independent variables $x^{1}, \cdots, x^{n}$, it can be supposed that (49) holds.

If $m=n$, then $\left({ }^{*}\right)$ is trivial. Suppose, therefore, that $m<n$. Identify (7) with (43) in the following way: let $n, m ; x^{1}, \cdots, x^{n} ; s ; z^{1}, \cdots, z^{m}$ in (7) correspond to $m, n-m-1 ; x^{1}, \cdots, x^{m} ; x^{m+1} ; x^{m+2}, \cdots, x^{n}$ in (43). Thus $A=\left(p_{\mu \nu}\right)$, where $\mu, \nu=1, \cdots, m ;-b=\left(p_{1 m+1}, \cdots, p_{n m+1}\right) ; f=A^{-1} b$; and $C=\left(p_{\mu \tau}\right)$, where $\mu=1, \cdots, m$ and $\tau=m+2, \cdots, n$. Thus (II) and the lemma are applicable.

The mapping $\left(x^{1}, \cdots, x^{m} ; x^{m+1} ; x^{m+2}, \cdots, x^{n}\right) \rightarrow\left(v^{1}, \cdots, v^{m} ; x^{m+1}\right.$, $\left.x^{m+2}, \cdots, x^{n}\right)$, corresponding to $(x ; s ; z) \rightarrow(v ; s ; z)$, transforms the forms $\omega$ (that is, (43)) into the forms (9) in $d v^{1}, \cdots, d v^{m}, d x^{m+2}, \cdots, d x^{n}$ with coefficients depending continuously on $v^{1}, \cdots, v^{m}, x^{m+1}, x^{m+2}, \cdots, x^{n}$.

If $m=n-1$, the proof is complete (with $y=\left(v^{1}, \cdots, v^{m}, x^{m+1}\right)$ ). If $m<n-1$, the procedure can be applied again, with the initial value problem (6) replaced by (50) and $v(0)=u$, where $z^{M}=x^{m+2}$ and $u$ is a (new) $m$-vector. According to the lemma, the right side of (50) does not depend on $s$, and, therefore, the solution $v\left(z^{M}\right)=v\left(u ; z^{M} ; x^{m+3}, \cdots, x^{n}\right)$ is independent of $s=x^{m+1}$. Hence, the inverse mapping $\left(s ; v ; z^{M} ; x^{m+3}, \cdots, x^{n}\right) \rightarrow\left(s, u ; z^{M}\right.$; $\left.x^{m+3}, \cdots, x^{n}\right)$ transforms (9) into linear forms in $d u^{1}, \cdots, d u^{m}, d x^{m+3}, \cdots$, $d x^{n}$ (with coefficients depending continuously on $\left(u^{1}, \cdots, u^{m}, x^{m+1}, x^{m+2}\right.$, $\left.\left.\cdots, x^{n}\right)\right)$. If $m=n-2$, this completes the proof of $\left({ }^{*}\right)$, with $y=\left(u^{1}, \cdots, u^{m}\right.$, $\left.x^{m+1}, \cdots, x^{n}\right)$. It is clear that this procedure can be continued to prove $\left(^{*}\right)$ for any $m(\leqq n)$.

\section{REFERENCES}

1. E. Cartan, Leçons sur les invariants intégraux, Paris, 1922.

2. C. Caratheodory, Variationsrechnung und partielle Differentialgleichungen erster Ordnung, Leipzig and Berlin, 1935. 
3. P. Gillis, Sur les equations linéaires aux differentielles totales, Bull. Soc. Roy. Sci. Liège vol. 9 (1940) pp. 197-212.

4. P. Hartman, On the local uniqueness of geodesics, Amer. J. Math. vol. 72 (1950) pp. 723730.

5. - On geodesic coordinates, ibid. vol. 73 (1951) pp. 949-954.

6. - On integrating factors and on conformal mappings, Trans. Amer. Math. Soc. vol. 87 (1958) pp. 387-406.

7. P. Hartman and A. Wintner, On the fundamental equations of differential geometry, Amer. J. Math. vol. 72 (1950) pp. 757-774.

8. - On the problems of geodesics in the small, ibid. vol. 73 (1951) pp. 132-148.

9. - Regular binary Pfaffians and nonparabolic partial differential equations, Rend. Circ. Mat. Palermo ser. 2, vol. 3 (1954) pp. 347-362.

10. A. Ostrowski, Sur un théorc̀me fondamental de la thêorie des équations linéaires aux dérivées partielles, Comment. Math. Helv. vol. 15 (1942-1943) pp. 217-221.

11. H. Weyl, Ueber die Bestimmung einer geschlossenen konvexen Fläche durch ihr Linienelement, Vierteljschr. Naturf. Ges. Zürich vol. 61 (1916) pp. 40-72.

The Johns Hopkins University, BALTIMORE, MD. 